\title{
Evolution of the external quantum efficiency of Si-based PV minimodules with encapsulated down-shifters and aged under UV radiation
}

\author{
Benjamín González-Díaz1, Min Hsian Saw2, Cecilio Hernández-Rodríguezz, Joaquín \\ Sanchiz4, Yong Sheng Khoo2, and Ricardo Guerrero-Lemus3*. \\ 1Departamento de Ingeniería Industrial. Universidad de La Laguna. Avenida Astrofísico \\ Francisco Sánchez S/N 38206 S/C de Tenerife. Spain. \\ 2Solar Energy Research Institute of Singapore (SERIS), National University of \\ Singapore (NUS), Singapore 117574, Singapore. \\ 3Departamento de Física. Universidad de La Laguna. Avenida Astrofísico Francisco \\ Sánchez S/N 38206 S/C de Tenerife. Spain. Email: rglemus@ull.edu.es \\ 4Departamento de Química. Universidad de La Laguna. Avenida Astrofísico Francisco \\ Sánchez S/N 38206 S/C de Tenerife. Spain.
}

\begin{abstract}
:
For the first time to our knowledge, a study about the increase in external quantum efficiency (EQE) by down-shifters (DS) encapsulated by industrial procedures on photovoltaic (PV) modules is presented. EQE improves substantially in the UV range not only because of the optical path optimization for photons reaching the solar cell, but also it is attributed to the substantial reduction of DS photons scattered out of the solar cell when the outermost surface of the DS layer is embedded in ethyl-vinyl-acetate. Consequently, a proper encapsulation of DS layers is required for the optimal characterization of EQE. Moreover, the encapsulation of the DS layer substantially reduces the degradation of its luminescent properties due to UV radiation. However, the reduction of the EQE under UV radiation for the encapsulated DS layer is still high and should be substantially reduced for the integration of down-shifting properties in the lamination process of PV modules.
\end{abstract}

Keywords: lanthanides, down-shifters, aging, UV radiation, luminescence.

Declarations of interest: none.

1.- Introduction

Nowadays the photovoltaic (PV) energy continues being one of the most interesting renewable energies to be implemented in the electrical systems, as the annual new solar PV system installations have risen from $29.5 \mathrm{GW}$, in 2012, to $480 \mathrm{GW}$ worldwide in 2018 [1]. The PV market is dominated by the silicon technology, representing a $95 \%$ of the 
market share [2]. Nonetheless, the main challenge of the Si-based PV industry to increase the conversion efficiency of the solar cells at the lowest cost still remains.

The maximum theoretical efficiency of a single junction solar cell was calculated through a detailed balance model by Shockley and Queisser in 1961, achieving a 33\% limit and $31 \%$ limit for a Si-based solar cell [3]. However, this limit can be theoretically exceeded by means of a broad range of technologies which implies the use of new materials and the spectral modification of the incoming light, moving beyond the first generation of Sibased solar modules [4]. This route can be cost-effective [5] and it is based on increasing the external quantum efficiency (EQE) of the device in the UV range. The process consists of down-converting (DC) or down-shifting (DS) the incident UV photons from the solar radiation to lower energy radiation in the visible range, where the EQE of the solar cells is closer to $1[6]$.

For DC processes, a single high-energy photon interacting with the DC layer splits its energy between two photons with longer wavelength. Then, when an optimal DC layer is used on a Si-based solar cell, the maximum theoretical solar cell's efficiency varies from $30.9 \%$ to $38.6 \%$ [7]. Contrariwise, DS is a process where photons shift the energy from a high-energy range, typically UV, into a lower energy range [5,6]. However, the DS process will not be able to overtake the Shockley-Queisser limit because no additional photons are generating electron-hole pairs $[4,8]$.

In recent years, a broad range of compounds and DS fabrication techniques have been analyzed in the literature [6,9-13], where rare earth compounds highlight due to its absorption properties in the UV range and emission in the visible range. In the case of Eu3+ complexes embedded in PMMA and applied to a standard silicon PV cell by different techniques $[4,6,8,14-16]$, we have recently obtained an enhancement of 0.31 percentage points in the conversion efficiency of a standard Si-based solar cell [17]. The DS film also showed excellent thermal stability supporting its application in the Si-based module assembly steps.

Different approaches have been studied and reported in the literature in order to design the most suitable and cost-effective procedure to place the DS in the PV device, characterize and maximize the converting properties. They can be classified in two groups: (i) DS integrated in the solar cell; and (ii) DS located in a separate layer on top of the device. However, as the diffusion of rare earths in the solar cell is very difficult and the luminescence obtained is very low [18], the best option is to place the DS on the PV device [17]. For this option, there have been works showing the addition of the DS as a dopant in the antireflection coating of the solar cell [19], but the most common procedure is to embed the DS in a polymer and directly apply it to the solar cell [20-22]. In a more refined procedure, the DS and PV cell were encapsulated between two borosilicate float glasses using a vacuum laminator [23]. However, placing the DS embedded in a polymer directly on the solar cell makes the experimental procedure difficult for comparing results, as each DS is fixed on a defined area of a device and the optoelectronic properties can 
vary between different regions of the solar cell surface and DS surface. Then, a more versatile alternative is to deposit the DS embedded in the polymer on a glass substrate and, then, place it on the solar cell [5]. However, an air gap is located between the DS on a glass substrate and the solar cell that reduces the EQE of the system. To avoid this gap, a matching oil can be located between the DS on glass and the solar cell to enhance the optical coupling and to avoid refractive index mismatching losses [24].

Moreover, as we have described previously, analyzing the effect of the integration of a DS in a spectral range where the EQE of the bare PV device is not negligible can be very difficult because the EQE of the PV device integrating the DS is the result of the two competing efficiencies (the DS and the bare PV cell) and the transmittance of the DS layer [25]. This makes difficult to analyze the results of the only scientific paper detected in the literature where a DS is encapsulated to a PV cell using a procedure close to the standard industrial process because the EQE of the PV cell encapsulated with clear ethylene vinyl acetate (EVA) is above $19 \%$ in the $300-400 \mathrm{~nm}$ spectral range [23]. Also, these authors use a glass backsheet to allow for transmission measurements, making the results not totally comparable to the ones expected for a standard PV module (and the DS is based on commercial dyes, instead of rare earths).

Regarding to the PV related fabrication normative, the standard IEC 61215 [26] establishes the durability and performance tests to assure the 25 years operational lifetime of Si-based PV modules. However, the first study for Eu3+ complex embedded in PMMA DS layer exposed to ambient conditions [27] showed a strong degradation of the complex when it was placed directly on top of the solar module. In contrast, when the DS layer was covered by a low-iron glass, there was no significant evidence of degradation in the EQE enhancement during the testing period (21 days) [27]. These experiments were made by depositing the DS layer on a glass substrate and placing the sample directly on top of the module, without any additional encapsulation process that could optimize the light optical path and minimize long term degradation against environmental conditions. The durability of the DS layers exposed to ambient conditions can be affected by several external agents such as radiation, humidity, dust, pollution, among others. Also, the climatology plays an important role on the degradation of the polymers and encapsulants [28] and, consequently, on the Si-based PV module performance [29].

In this work, for the first time to our knowledge, a study about the increase in EQE of DS/glass samples encapsulated on PV modules using standard industrial procedures is presented. The encapsulation process not only assures a more robust protection against ambient conditions than previously published [27], but also provides a more efficient path for the incident light reaching the PV cell, as it will be demonstrated below. Moreover, in this work, the ageing of the devices is controlled and accelerated in a climatic chamber for a more precise description of the impact of UV radiation on the stability of Eu3+ complex embedded in PMMA DS layer in increasing conversion efficiency. This work is 
an extension of a recent work showing an alternative and fully experimental procedure for characterizing DS placed on PV devices [25].

\section{2.- Experimental}

\subsection{Down-shifter fabrication}

The fabrication of the DS layers has been carried out from europium(III) nitrate pentahydrate (99.99\%), 4,7-biphenyl-1,10-phenanthroline (bphen, 97\%), thenoyltrifluoroacetone (Htta, 99\%), triethylamine (99\%), poly(methylmethacrylate), (PMMA, average Mw 996,000, ref. 182265) from Aldrich. All the reagents have been used as received.

The DS layers are prepared in two stages, first the europium complex [Eu(tta)3(bphen)] is prepared and then it is embedded in the PPMA film. For the preparation of the active species, Htta (666 mg, $3 \mathrm{mmol})$ was dissolved in ethanol $(10 \mathrm{~mL})$ and triethylamine (418 $\mu \mathrm{L}, 3 \mathrm{mmol}$ ) was added and the solution stirred at $60^{\circ} \mathrm{C}$ for 15 minutes. After that, bphen, $(332 \mathrm{mg}, 1 \mathrm{mmol})$ in ethanol $(2 \mathrm{~mL})$ was added. Finally, $\mathrm{Eu}\left(\mathrm{NO}_{3}\right)_{3} \cdot 5 \mathrm{H}_{2} \mathrm{O}(428 \mathrm{mg}, 1$ $\mathrm{mmol})$ in ethanol $(1 \mathrm{~mL})$ was added dropwise.

The mixture was maintained at $60^{\circ} \mathrm{C}$ during $3 \mathrm{~h}$. After this time, $10 \mathrm{~mL}$ of water were added and the precipitate was filtered and washed with water. The crude product was recrystallized from $95 \%$ ethanol. Yield $\sim 80 \%$. Elemental analysis calculated $(\%)$ for $\mathrm{C}_{48} \mathrm{H}_{28} \mathrm{~N}_{2} \mathrm{EuOO}_{6} \mathrm{~F}_{9} \mathrm{~S}_{3}$ : C, 50.23; H, 2.46; N, 2.44; S, 8.38. obtained (\%): C, 50.47; H, 2.47; $\mathrm{N}, 2.69 ; \mathrm{S}, 8.51$. The elemental analysis is consistent with the molecular structure published in [27].

Therefore, $42.0 \mathrm{mg}$ of the active species was dissolved in $21 \mathrm{~mL}$ of $\mathrm{CH} 2 \mathrm{Cl} 2$ in a refrigerated ultrasonic bath. Subsequently, $210 \mathrm{mg}$ of PMMA were added and the mixture stirred until the complete solution of the polymer. The solution was spin-coated on top of low iron glasses. The homogeneity of the DS layer for the different samples was also checked. The solvent was evaporated at room temperature. Samples with $20 \%$ relative weight concentration of the active species with respect to the PMMA were obtained [25].

\subsection{Description of the used minimodules}

To perform the ageing experiments of the DS layers, a PV minimodule was designed and fabricated. A standard $156 \mathrm{~mm}$ x $156 \mathrm{~mm}$ multicrystalline silicon solar cell was encapsulated in a conventional structure composed by low iron glass with Cerium Oxide antireflection coating, EVA, solar cell, EVA and Tedlar-polyester-Tedlar backsheet. The electrical connections were created by soldering bus ribbons onto the solar cell and interconnecting them with a $5 \mathrm{~mm}$ cross-connector. Before the DS layer emplacement, the solar minimodule has been characterized in the laboratory according to IEC 61853 part 
one and part two [30,31]. We intentionally used a solar cell with efficiency below the state of the art because we need a near zero EQE in the spectral range where the DS is produced to avoid any competing effect from the intrinsic EQE of the mc-Si cell and, therefore, to obtain a clean value of the encapsulation effect of the DS layer.

\subsection{Incorporation of the DS layers on the solar minimodules}

In order to analyze the degradation of the DS layers, three experimental configurations have been developed. In the first configuration, the DS deposited on the low iron glass is placed on the PV minimodule with the DS layer directly exposed to the atmosphere (Fig. 1a). In the second configuration, the DS layer is in direct contact with the top glass of the PV minimodule (Fig. 1b). Finally, in the third configuration, the DS layer is in contact with an EVA sheet placed on the PV minimodule and, then, encapsulated to the PV minimodule by applying a standard industrial lamination process (Fig. 1c). In this lamination process the laminate is heated under controlled temperature-pressure-time profile in a laminator equipment to form a multi-layer laminate. The process usually follows four steps, which may overlap. The first step is the heating of the laminate for cross-linking at $145^{\circ} \mathrm{C}$ during the whole process. In the second step, the air voids are removed under low-pressure ( $<1$ mbar). Then, in the third step, a high pressure membrane is applied to the top of the laminate. Lastly, the fourth step is the final cooling, where natural air cooling is used.

The used encapsulant is EVA. During the curing process, the EVA is converted into a cross-linked thermosetting material which creates the protective layer of the DS layer in the PV minimodule. This layer provides mechanical support, electrical isolation, temperature stresses and protection against environmental elements.

\section{a) DS layer face up}

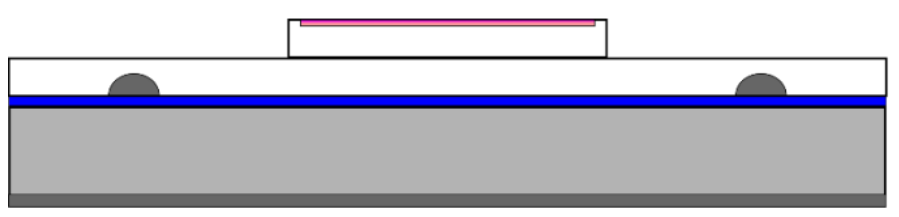

b) DS layer face down
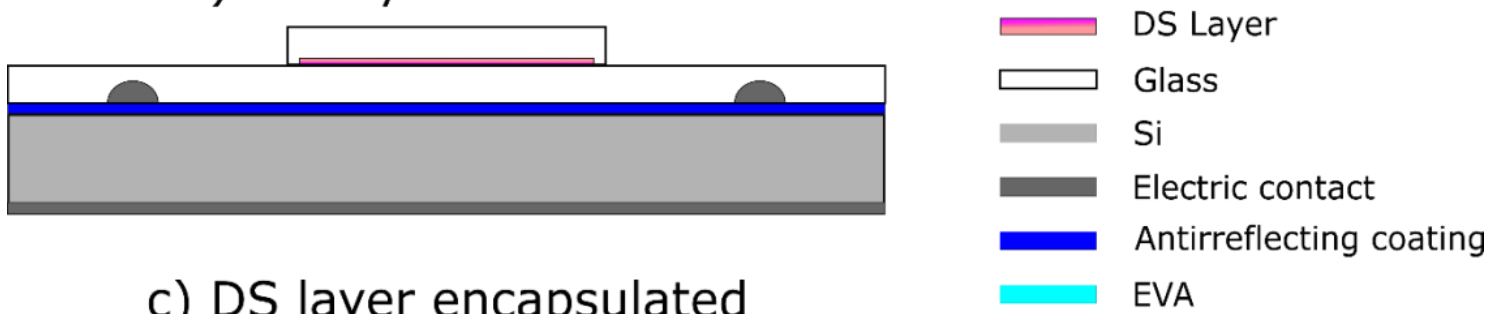

c) DS layer encapsulated

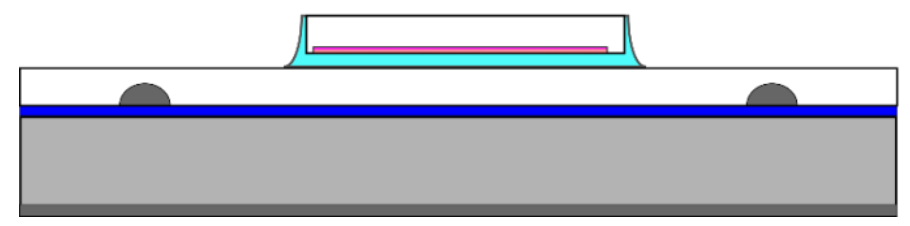


Fig. 1.- The three configurations of PV minimodule with integrated DS layers that are studied in this work.

\subsection{Characterization setup}

To evaluate the UV degradation of the DS layers, a Xenoclima Chamber model 1500RF manufactured by CCI has been used, equipped with a $150 \mathrm{~W}$ xenon lamp. In Fig. 2 the spectrum of the lamp is plotted. To evaluate the UV dose provided by this climatic chamber to our device, the amount of irradiation in a defined UV range has been calculated and compared to the solar spectrum. Indeed, the total amount of irradiance of the $150 \mathrm{~W}$ xenon lamp in the range $350-400 \mathrm{~nm}$ is $227.47 \mathrm{~W} / \mathrm{m} 2$, approximately 2.4 times higher than the AM1.5G irradiance at the same spectral range.

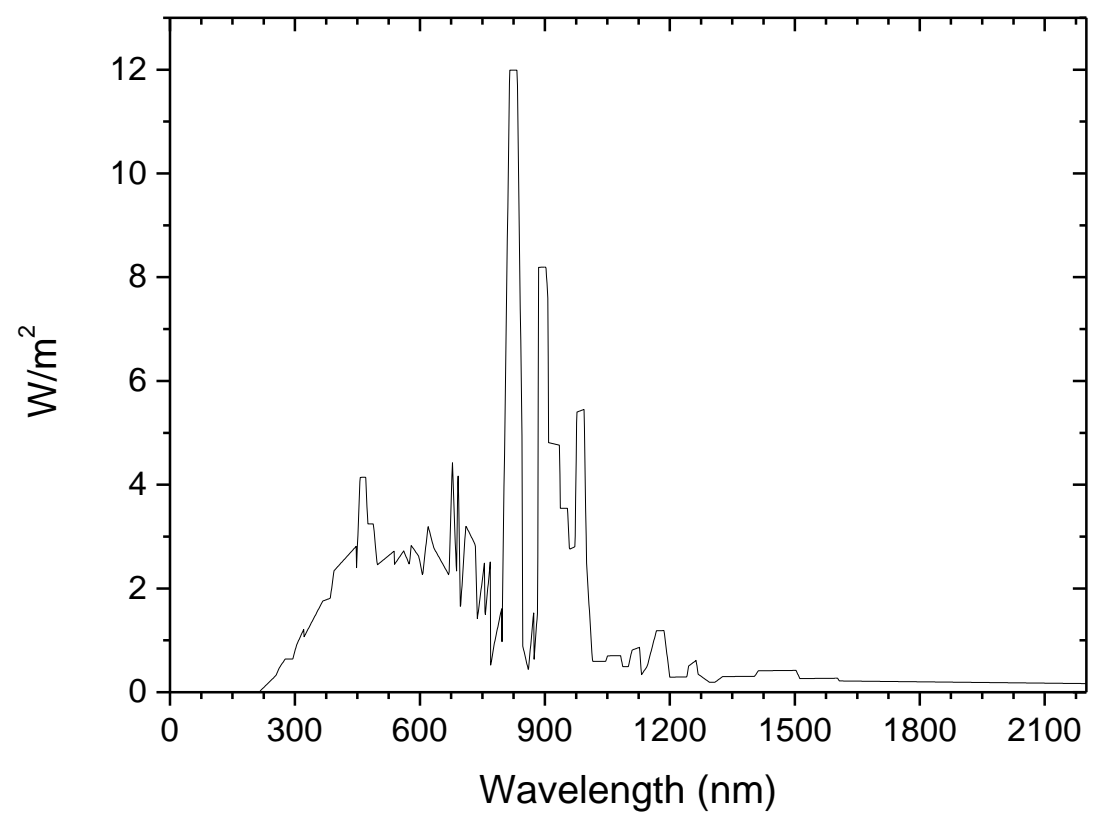

Fig. 2.- Irradiance spectra of the $150 \mathrm{~W}$ xenon lamp of the climatic chamber used in this work.

The temperature inside of the climatic chamber can be adjusted in the range $10^{\circ} \mathrm{C}-70^{\circ} \mathrm{C}$ and the relative humidity $(\mathrm{RH})$ can be adjusted from 0 to $100 \%$. In all the ageing experiments in order to analyze the UV degradation of the device, the temperature has been adjusted to $21^{\circ} \mathrm{C}$ and the RH to $20 \%$.

The EQE measurements in the 280-1200 nm wavelength range have been acquired using a small beam spectral response measurement system (IVT Solar, PVE-300). Spot area illumination of the system allows localized measurements and hence provides accurate comparison. Spot area EQE measurements were first performed on a pre-defined spot of the bare PV minimodule. Subsequently, DS/glass substrate was placed on the same spot 
of the PV module and sample's EQE were measured. This allows an accurate comparison of the module EQE with and without DS layer in the UV range.

\section{3.- Results and discussion}

The PV minimodule with the DS layers was analyzed prior to the ageing process. The measured EQE spectra are shown in Fig. 3 ranging from 300 to 1200 and in the range from 300 to $355 \mathrm{~nm}$. Also, the EQE of CIGS [32] and mc-Si [33] modules with the highest independently confirmed efficiencies are exposed.
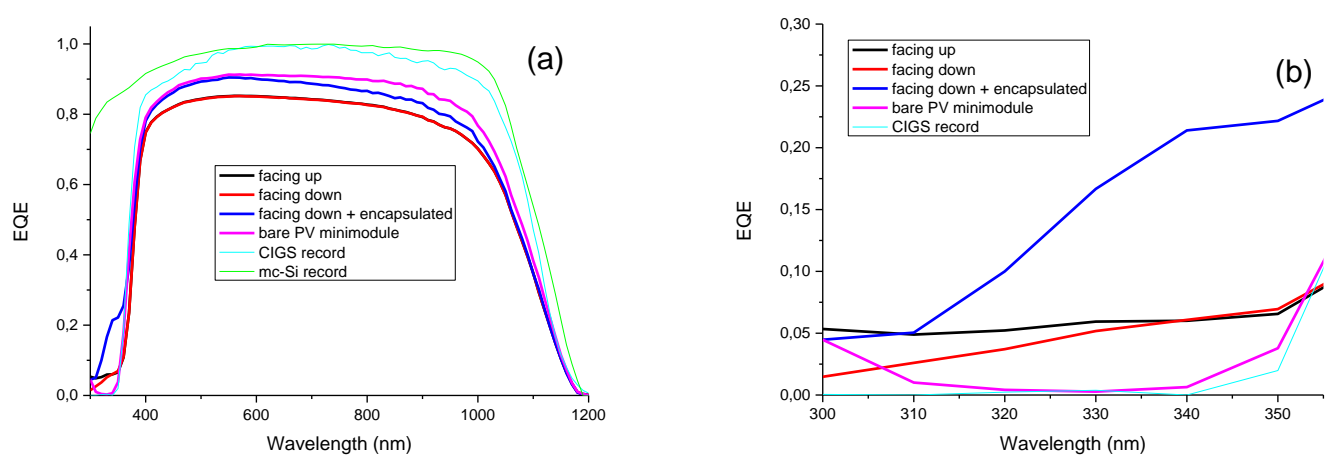

Fig. 3.- EQE of the PV minimodule: (i) bare PV minimodule; (ii) with a DS layer encapsulated; (iii) with a DS layer faced down; and (iv) with a DS layer faced up in contact with the atmosphere. EQEs in the (a) $300-1200 \mathrm{~nm}$ and (b) $300-355 \mathrm{~nm}$ spectral ranges.

The samples with the DS layers show larger EQE in the UV spectral range compared to the bare PV minimodule. According to the photoluminescence previously reported [6,34], the maximum luminescent peak of the DS layers is centered around $615 \mathrm{~nm}$. The sample with the DS layer encapsulated presents the largest increase in EQE reaching values above $20 \%$ at $340 \mathrm{~nm}$ and longer wavelengths. Of course, of the bare PV minimodule is the highest at wavelengths higher to the range where the downshifting of UV photons is produced because it has no losses due to any additional glass/PMMA sample on top.

In the UV range between $300 \mathrm{~nm}$ and $355 \mathrm{~nm}$, if we normalize the EQE of the device with the encapsulated DS layer minus the EQE of the bare PV minimodule, the analysis shows that the EQE when the DS layer is non-encapsulated and facing down is only $25.51 \%$ and when the non-encapsulated DS layer is facing up is $35.88 \%$ of the EQE of the PV minimodule with the encapsulated DS layer. In the range $355-1200 \mathrm{~nm}$, the EQE results of the DS layers faced up and faced down in this range are $95.11 \%$ and $95.02 \%$, respectively, of the encapsulated DS layer.

Therefore, the improvement in conversion efficiency for the encapsulated DS layer is interpreted by two main mechanism. First, the obtained EQE values are higher not only 
in the UV range, but in the complete EQE spectral range partially due to the encapsulated configuration avoids the air gap between the DS layer and the minimodule. In this case, if we approximate for EVA, PMMA and the low-iron glass the same and average refraction indexes of 1.5, the expected transmittance because of the air gap and using the simplest Fresnel eq. for normal incidence $\left(\left[n_{1}-n_{2}\right] 2 /\left[n_{1}+n_{2}\right]_{2}\right)$ is $92.16 \%$, quite consistent with the losses in EQE exposed above for the 355 - $1200 \mathrm{~nm}$ spectral range. However, this approximation is not valid in the $300-355 \mathrm{~nm}$ spectral range because of the substantial increase in EQE shown for the device with the DS layer encapsulated. We attribute this substantial increase to the fact that the encapsulation fills with EVA all the voids and roughness of the DS layer surface spin-coated, as we have previously characterized by AFM [35], and between the DS layer and the low-iron glass of the minimodule. Literature reports Rayleigh scattering within the polymers due to different structural irregularities [36]. Then, the roughness of the DS layer not encapsulated produces light scattering of the DS photons, enhanced at shorter wavelengths and, consequently, reducing the amount of the DS photons that reaches the solar cell, hence resulting in less enhancement of the EQE of the device. Also, this effect could be attributed to a larger mismatch between refraction indexes for EVA, PMMA and low-iron glass at $300-355 \mathrm{~nm}$, but the opposite is reported in the scientific literature $[37,38]$.

In addition, the encapsulated layer presents greater efficiency and also greater durability. The two effects can be explained to some extent by lower contact of the europium complex with atmospheric oxygen in the encapsulated layer. The oxygen molecule has a triplet ground state which deactivates the triplet excited states of the ligands involved in the down-Shifting process and therefore the intensity of the photoluminescence is decreased. As a result of the deactivation, the triplet oxygen transits to highly reactive singlet oxygen [39]. And for the case of the compounds that concern us, the thienyl groups in the tta ligand are sensitive to the singlet dioxygen and they may suffer irreversible oxidation which results in progressive destruction of the active species and therefore in a continuous decrease of the emission intensity [40]. Polymeric films with low oxygen permeability, such as PMMA, have a limited protective effect [41,42], but the encapsulation and the additional protection provided by the glass and the EVA improves the emission intensity, increases the durability of the complex and maintains its performance for a longer time.

The EQEs for mc-Si and CIGS modules with record efficiencies are added to fig. 3 to show that DS layers on high efficient c-Si and mc-Si solar modules can be detrimental for enhancing the efficiency of such devices, as we have explained elsewhere [25]. However, the high UV transparency of such devices could accelerate the passivation degradation of the solar cell, as it has been described in recent works $[43,44]$. Then, the DS can be also useful for absorbing UV radiation and, therefore, to slow the passivation degradation reported. Also, the DS layers can be very useful to enhance the efficiency of other PV technologies as in highly efficient CIGS modules, as it can also be appreciated in fig. 3. 
Subsequently, all the samples were subjected to the ageing process at the same time. To analyze the degradation of the DS layers, the RH was set at $20 \%$ and the chamber temperature was adjusted to $21^{\circ} \mathrm{C}$, to isolate the UV effects on the layers, in contraposition to the experiments reported in outdoor conditions, where several degradation mechanisms (e.g. UV irradiation and humidity) act simultaneously [27]. To compare the performance of the DS layers, the contribution of the bare minimodule was subtracted and the EQE values from 300 and $380 \mathrm{~nm}$ were integrated and compared. After several cycles, the drop in EQE due to UV degradation of the DS layers placed on top of the minimodule faced up and faced down without encapsulation started being detected, as it is shown in fig. 4.

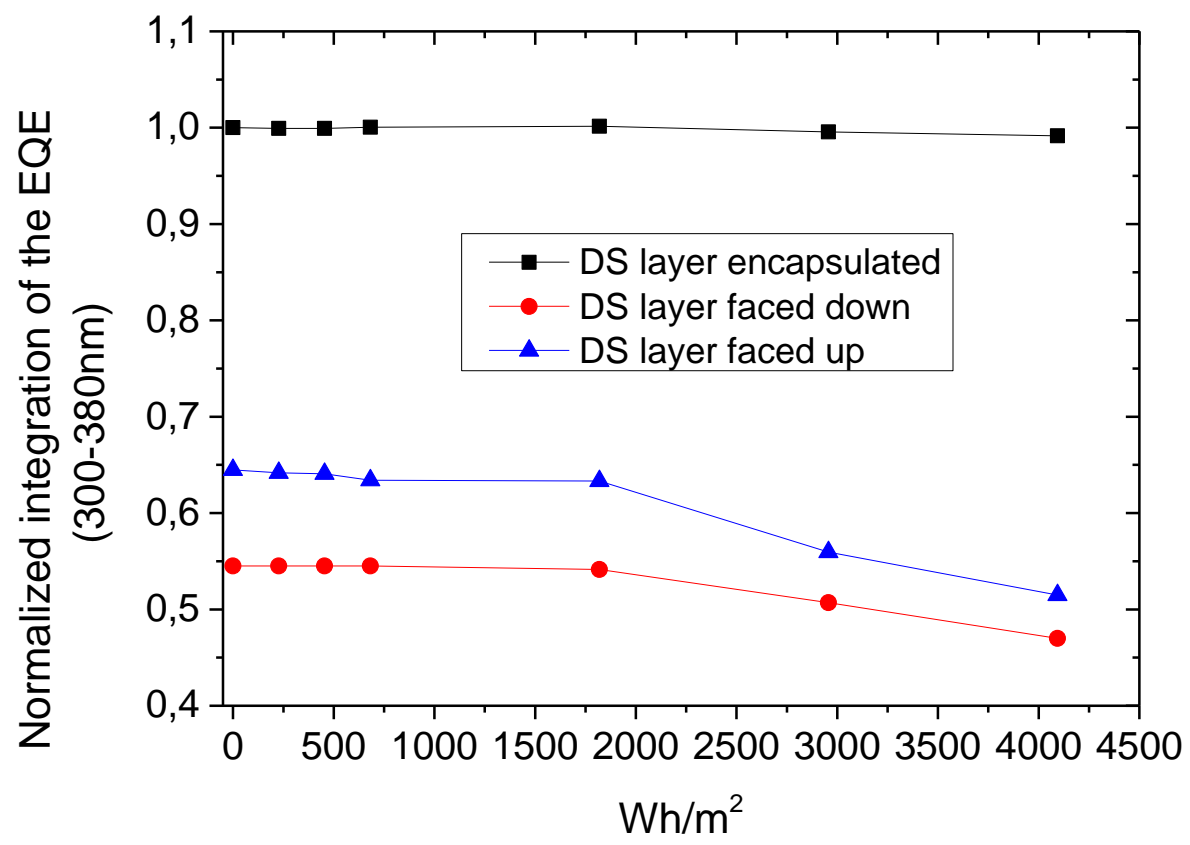

Figure 4.- Integrated increase in EQE values in the range $300-380 \mathrm{~nm}$ for the DS layers subjected to UV degradation in the climatic chamber.

After a UV dose of $4093 \mathrm{Wh} / \mathrm{m}_{2}$ in the climatic chamber at $20^{\circ} \mathrm{C}$ and $20 \% \mathrm{RH}$, the samples directly placed on top of the minimodule show a remarkable degradation in comparison with the encapsulated DS layer on the PV minimodule. These differences can be quantified in terms of the slope estimated for each curve in fig. 4 (Fig. 5). These results reveal that the encapsulation prevents against the rapid degradation of the DS layer placed on PV minimodules and exposed to ambient conditions. Moreover, the obtained results of the sample faced up initially are slightly higher than for the sample faced down. This result is attributed to the fact that the glass substrate absorption is dependent on the wavelengths of the incident photons, as we have described elsewhere [25]. However, the slope observed under UV degradation is higher for the DS facing up directly to the UV light than for the sample with the DS facing down. This difference is attributed to the fact 
that the glass substrate of the DS facing down is partially protecting the DS layer against the UV radiation.

The model reveals that the DS layer faced down is degraded at about $18 \mathrm{kWh} / \mathrm{m} 2 \mathrm{UV}$ irradiation, and around $17 \mathrm{kWh} / \mathrm{m} 2 \mathrm{UV}$ irradiation for the sample with the DS layer faced up. In contrast, the sample with the DS layer encapsulated shows a $20.6 \%$ reduction of the EQE integrated value after a $60 \mathrm{kWh} / \mathrm{m} 2$ UV dose. In a projection of the UV degradation of the encapsulated DS layer, the total extinction of the DS properties is expected to take place at about $300 \mathrm{kWh} / \mathrm{m} 2$. If we consider an average 5 equivalent sun hours per day, this UV dose is equivalent to about 4.3 months of outdoor exposure. This value is still very low as compared to the estimated 20-25 years operational lifetime of PV module. It remains challenging if encapsulated DS layers aspire to be integrated in PV modules for real world applications.

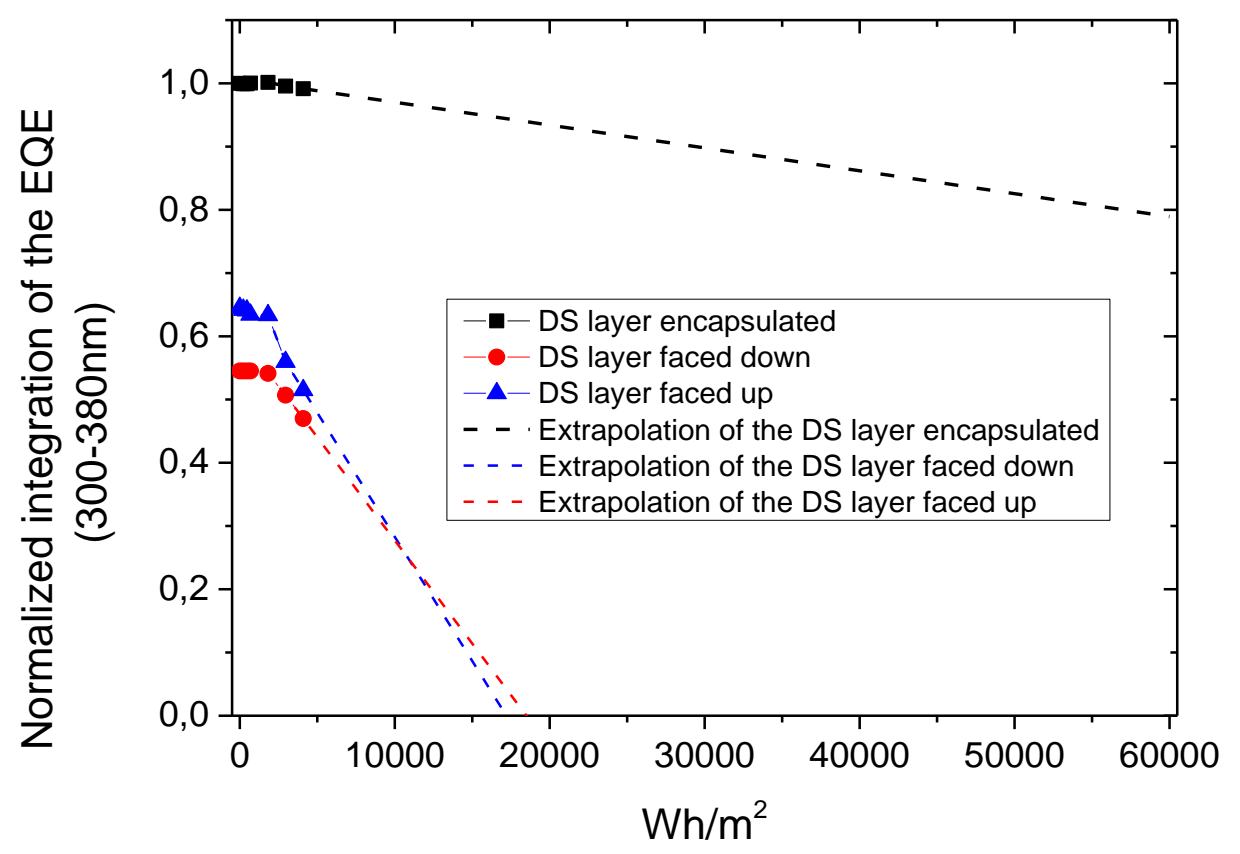

Figure 5.- Linear degradation expected for the different DS layers on the PV minimodule and subjected to UV irradiation.

\section{Conclusions}

In this work, the UV degradation of DS layers in terms of the evolution of the EQE of the PV minimodule where these DS layers are placed has been reported. Three different configurations have been evaluated: (i) a DS layer on top of the PV minimodule with the converter directly exposed to the ambient conditions; (ii) a DS layer on top of the PV 
minimodule with the converter in touch with the solar module; and (iii) a DS layer with the converter in touch with the PV minimodule and encapsulated.

The results show that the non-encapsulated DS layers present much lower EQE values in the $300-355 \mathrm{~nm}$ spectral range compared to the device with the encapsulated DS layer. This effect is attributed to the avoidance of any air gap between the DS sample and the PV minimodule, but also to the scattering of the DS photons out of the solar cell when the DS layer is not encapsulated in EVA. Therefore, a proper encapsulation of DS layers is required for the optimal characterization of EQE.

Also, these non-encapsulated samples experiment major UV degradation of the DS properties compared to the encapsulated DS layer. However, the stability of the EQE measured for the encapsulated DS layer placed on the PV minimodule should be improved substantially for integrating these DS layers in PV modules for actual applications.

The encapsulated configuration of the DS layer partially decreases the absorption of the UV radiation by the solar module and therefore reduces the thermalization losses in the solar cell and the photodegradation of the polymeric layers. Both effects enhance the lifetime of the solar panel and optimize the energy production, allowing better performance of the PV panels.

The analysis of other active species and polymers are in progress in order to increase the stability and efficiency of the DS layers.

Acknowledgement

This work has been financed by the Ministerio de Ciencia, Innovación y Universidades, Spain (RTI2018-095563-B-100), co-supported by the European Social Fund. The work at Solar Energy Research Institute of Singapore (SERIS) was sponsored by the National University of Singapore (NUS) and Singapore's National Research Foundation (NRF) through the Singapore Economic Development Board (EDB).

\section{Data availability}

The processed data required to reproduce these findings are available to download from the Mendeley data repository.

\section{References}

[1] IRENA Resource, Data and Statistics - Dashboard, (n.d.). 
http://resourceirena.irena.org/gateway/dashboard/?q=photovoltaic\& topic=4\&sub Topic $=16$ (accessed June 1, 2019).

[2] Fraunhofer ISE, Photovoltaic Report, (2018). https://www.ise.fraunhofer.de/content/dam/ise/de/documents/publications/studies /Photovoltaics-Report.pdf (accessed June 1, 2019).

[3] W. Shockley, H.J. Queisser, Detailed Balance Limit of Efficiency of p-n Junction Solar Cells, J. Appl. Phys. 32 (1961) 510. https://doi.org/10.1063/1.1736034.

[4] J. Day, S. Senthilarasu, T.K. Mallick, Improving spectral modification for applications in solar cells: A review, Renew. Energy. 132 (2019) 186-205. https://doi.org/10.1016/j.renene.2018.07.101.

[5] T. Monzón-Hierro, J. Sanchiz, S. González-Pérez, B. González-Díaz, S. Holinski, D. Borchert, C. Hernández-Rodríguez, R. Guerrero-Lemus, A new cost-effective polymeric film containing an $\mathrm{Eu}(\mathrm{III})$ complex acting as UV protector and downconverter for Si-based solar cells and modules, Sol. Energy Mater. Sol. Cells. 136 (2015) 187-192. https://doi.org/10.1016/j.solmat.2015.01.020.

[6] S. González-Pérez, J. Sanchiz, V.D. Rodríguez, D. Cañadillas-Ramallo, J. González-Platas, D. Borchert, B. González-Díaz, C. Hernández-Rodríguez, R. Guerrero-Lemus, Highly luminescent film as enhancer of photovoltaic devices, J. Lumin. 201 (2018). https://doi.org/10.1016/j.jlumin.2018.04.038.

[7] T. Trupke, M.A. Green, P. Würfel, Improving solar cell efficiencies by downconversion of high-energy photons, J. Appl. Phys. 92 (2002) 1668-1674. https://doi.org/10.1063/1.1492021.

[8] J. Jiao, S. Gai, Y. Li, W. Shen, J. Tang, Y. Wang, L. Huang, J. Liu, W. Wang, L.A. Belfiore, $\mathrm{NaYbF} 4: \mathrm{Tb} / \mathrm{Eu}$ modified with organic antenna for improving performance of polymer solar cells, Electrochim. Acta. 260 (2018) 959-964. https://doi.org/10.1016/j.electacta.2017.12.077.

[9] T. Wang, B. Yu, Z. Hu, X. Wang, G. Zou, Q. Zhang, Enhancing the performance of multi-crystalline silicon photovoltaic module by encapsulating high efficient Eu3+ complex into its pre-existing EVA layer, Opt. Mater. (Amst). 35 (2013) 1118-1123. https://doi.org/10.1016/j.optmat.2012.12.031.

[10] X. Huang, S. Han, W. Huang, X. Liu, Enhancing solar cell efficiency: The search for luminescent materials as spectral converters, Chem. Soc. Rev. 42 (2013) 173201. https://doi.org/10.1039/c2cs35288e.

[11] X. Wang, T. Wang, X. Tian, L. Wang, W. Wu, Y. Luo, Q. Zhang, Europium complex doped luminescent solar concentrators with extended absorption range from UV to visible region, Sol. Energy. 85 (2011) 2179-2184. https://doi.org/10.1016/j.solener.2011.06.007.

[12] B. González-Díaz, B. Díaz-Herrera, R. Guerrero-Lemus, J. Méndez-Ramos, V.D. Rodríguez, C. Hernández-Rodríguez, J.M. Martínez-Duart, Erbium doped stain etched porous silicon, Mater. Sci. Eng. B Solid-State Mater. Adv. Technol. 146 (2008) 171-174. https://doi.org/10.1016/j.mseb.2007.07.020.

[13] B. Díaz-Herrera, B. González-Díaz, R. Guerrero-Lemus, C. Hernández-Rodríguez, 
J. Méndez-Ramos, V.D. Rodríguez, Photoluminescence of porous silicon stain etched and doped with erbium and ytterbium, Phys. E Low-Dimensional Syst. Nanostructures. 41 (2009) 525-528. https://doi.org/10.1016/j.physe.2008.09.015.

[14] X. Wang, Q. Yan, P. Chu, Y. Luo, Z. Zhang, S. Wu, L. Wang, Q. Zhang, Analysis on fluorescence of dual excitable Eu(TTA)3DPBT in toluene solution and PMMA, J. Lumin. 131 (2011) 1719-1723. https://doi.org/10.1016/j.jlumin.2011.03.061.

[15] H. Kataoka, T. Kitano, T. Takizawa, Y. Hirai, T. Nakanishi, Y. Hasegawa, Photoand thermo-stable luminescent beads composed of Eu(III) complexes and PMMA for enhancement of silicon solar cell efficiency, J. Alloys Compd. 601 (2014) 293297. https://doi.org/10.1016/j.jallcom.2014.01.165.

[16] M.B. de la Mora, O. Amelines-Sarria, B.M. Monroy, C.D. Hernández-Pérez, J.E. Lugo, Materials for downconversion in solar cells: Perspectives and challenges, Sol. Energy Mater. Sol. Cells. 165 (2017) 59-71. https://doi.org/10.1016/j.solmat.2017.02.016.

[17] R. Guerrero-Lemus, J. Sanchiz, M. Sierra-Ramos, I.R. Martín, C. HernándezRodríguez, D. Borchert, Downshifting maximization procedure applied to [Eu(bphen)(tta)3] at different concentrations applied to a photovoltaic device and covered with a hemispherical reflector, Sensors Actuators, A Phys. 271 (2018) 6065. https://doi.org/10.1016/j.sna.2018.01.010.

[18] B. Díaz-Herrera, E. Jiménez-Rodríguez, B. González-Díaz, A. MontesdeocaSantana, J.J. Velázquez, R. Guerrero-Lemus, Combined up conversion, down conversion and down shifting photo-luminescence of low cost erbium-ytterbium co-doped porous silicon produced by stain etching, Thin Solid Films. 519 (2011) 6110-6114. https://doi.org/10.1016/j.tsf.2011.04.029.

[19] I.S. Yu, S.C. Wu, L. Dumont, J. Cardin, C. Labbé, F. Gourbilleau, Monolithic crystalline silicon solar cells with $\mathrm{SiN} x$ layers doped with $\mathrm{Tb} 3+$ and $\mathrm{Yb} 3+$ rareearth ions, J. Rare Earths. 37 (2019) 515-519. https://doi.org/10.1016/j.jre.2018.07.014.

[20] S.K. Karunakaran, C. Lou, G.M. Arumugam, C. Huihui, D. Pribat, Efficiency improvement of Si solar cells by down-shifting Ce3+-doped and down-conversion Ce3+-Yb3+ co-doped YAG phosphors, Sol. Energy. 188 (2019) 45-50. https://doi.org/10.1016/j.solener.2019.05.076.

[21] L. de A. Florêncio, L.A. Gómez-Malagón, B.C. Lima, A.S.L. Gomes, J.A.M. Garcia, L.R.P. Kassab, Efficiency enhancement in solar cells using photon downconversion in $\mathrm{Tb} / \mathrm{Yb}$-doped tellurite glass, Sol. Energy Mater. Sol. Cells. 157 (2016) 468-475. https://doi.org/10.1016/j.solmat.2016.07.024.

[22] H. Yao, H. Shen, Q. Tang, Highly luminescent up/down conversion thin films prepared by a room temperature process, Thin Solid Films. 683 (2019) 1-7. https://doi.org/10.1016/j.tsf.2019.05.010.

[23] E. Klampaftis, B.S. Richards, Improvement in multi-crystalline silicon solar cell efficiency via addition of luminescent material to EVA encapsulation layer, Prog. Photovolt Res. Appl. 19 (2011) 345-351. 
[24] H. Lian, Z. Hou, M. Shang, D. Geng, Y. Zhang, J. Lin, Rare earth ions doped phosphors for improving efficiencies of solar cells, Energy. 57 (2013) 270-283. https://doi.org/10.1016/j.energy.2013.05.019.

[25] R. Guerrero-Lemus, J. Sanchiz, M. Sierra, I.R. Martín, C. Hernández-Rodríguez, D. Borchert, Alternative and fully experimental procedure for characterizing down-shifters placed on photovoltaic devices, Sol. Energy Mater. Sol. Cells. 185 (2018). https://doi.org/10.1016/j.solmat.2018.05.051.

[26] A. Omazic, G. Oreski, M. Halwachs, G.C. Eder, C. Hirschl, L. Neumaier, G. Pinter, M. Erceg, Relation between degradation of polymeric components in crystalline silicon PV module and climatic conditions: A literature review, Sol. Energy Mater. Sol. Cells. 192 (2019) 123-133. https://doi.org/10.1016/j.solmat.2018.12.027.

[27] B. González-Díaz, M. Sierra-Ramos, J. Sanchiz, R. Guerrero-Lemus, Durability analysis of the [Eu(bphen)(tta)3] down-shifter on Si-based PV modules exposed to extreme outdoor conditions, Sensors Actuators, A Phys. 276 (2018) 312-319. https://doi.org/10.1016/j.sna.2018.04.045.

[28] D.C. Miller, J.G. Bokria, D.M. Burns, S. Fowler, X. Gu, P.L. Hacke, C.C. Honeker, M.D. Kempe, M. Köhl, N.H. Phillips, K.P. Scott, A. Singh, S. Suga, S. Watanabe, A.F. Zielnik, Degradation in photovoltaic encapsulant transmittance: Results of the first PVQAT TG5 artificial weathering study, Prog. Photovoltaics Res. Appl. 27 (2019) 391-409. https://doi.org/10.1002/pip.3103.

[29] M. Schweiger, J. Bonilla, W. Herrmann, A. Gerber, U. Rau, Performance stability of photovoltaic modules in different climates, Prog. Photovolt Res. Appl. 25 (2017) 968-981. https://doi.org/10.1002/pip.2904.

[30] A. Sinha, O.S. Sastry, R. Gupta, Nondestructive characterization of encapsulant discoloration effects in crystalline-silicon PV modules, Sol. Energy Mater. Sol. Cells. 155 (2016) 234-242. https://doi.org/10.1016/j.solmat.2016.06.019.

[31] R. Eke, T.R. Betts, R. Gottschalg, Spectral irradiance effects on the outdoor performance of photovoltaic modules, Renew. Sustain. Energy Rev. 69 (2017) 429-434. https://doi.org/10.1016/j.rser.2016.10.062.

[32] M.A. Green, Y. Hishikawa, E.D. Dunlop, D.H. Levi, J. Hohl-Ebinger, A.W.Y. HoBaillie, Solar cell efficiency tables (version 52), Prog. Photovoltaics Res. Appl. 26 (2018) 427-436. https://doi.org/10.1002/pip.3040.

[33] M.A. Green, K. Emery, Y. Hishikawa, W. Warta, E.D. Dunlop, D.H. Levi, A.W.Y. Ho-Baillie, Solar cell efficiency tables (version 49), Prog. Photovoltaics Res. Appl. 25 (2017) 3-13. https://doi.org/10.1002/pip.2855.

[34] R. Guerrero-Lemus, J. Sanchiz, M. Sierra-Ramos, I.R. Martín, C. HernándezRodríguez, D. Borchert, Downshifting maximization procedure applied to $[\mathrm{Eu}(\mathrm{bphen})(\mathrm{tta})<\mathrm{inf}>3</ \mathrm{inf}>]$ at different concentrations applied to a photovoltaic device and covered with a hemispherical reflector, Sensors Actuators, A Phys. 271 (2018). https://doi.org/10.1016/j.sna.2018.01.010.

[35] S. González-Pérez, J. Sanchiz, B. González-Díaz, S. Holinski, D. Borchert, C. 
Hernández-Rodríguez, R. Guerrero-Lemus, Luminescent polymeric film containing an $\mathrm{Eu}(\mathrm{III})$ complex acting as $\mathrm{UV}$ protector and down-converter for $\mathrm{Si}$ based solar cells and modules, Surf. Coatings Technol. 271 (2015) 106-111. https://doi.org/10.1016/j.surfcoat.2014.12.074.

[36] T. Kaino, Encyclopedia of Polymeric Nanomaterials, Encycl. Polym. Nanomater. (2021) 1-14. https://doi.org/10.1007/978-3-642-36199-9.

[37] K.R. McIntosh, J.N. Cotsell, J.S. Cumpston, A.W. Norris, N.E. Powell, B.M. Ketola, An optical comparison of silicone and EVA encapsulants for conventional silicon PV modules: A ray-tracing study, in: 2009 34th IEEE Photovolt. Spec. Conf., $\quad$ IEEE, 2009: pp. 000544-000549. https://doi.org/10.1109/PVSC.2009.5411624.

[38] K.-C.D. Cheng, M.-L. V. Tse, G. Zhou, C.-F.J. Pun, W.-K.E. Chan, C. Lu, P.K. Wai, H. Tam, Optimization of 3-hole-assisted PMMA optical fiber with double cladding for UV-induced FBG fabrication, Opt. Express. 17 (2009) 2080. https://doi.org/10.1364/OE.17.002080.

[39] M.A. Filatov, S. Baluschev, K. Landfester, Protection of densely populated excited triplet state ensembles against deactivation by molecular oxygen, Chem. Soc. Rev. 45 (2016) 4668-4689. https://doi.org/10.1039/c6cs00092d.

[40] H. Kataoka, S. Omagari, T. Nakanishi, Y. Hasegawa, Photo-degradation analysis of luminescent polymers with lanthanide complexes, J. Photopolym. Sci. Technol. 28 (2015) 247-254. https://doi.org/10.2494/photopolymer.28.247.

[41] Y. Amao, I. Okura, T. Miyashita, Optical oxygen sensing based on the luminescence quenching of europium(III) complex immobilized in fluoropolymer film, Bull. Chem. Soc. Jpn. 73 (2000) 2663-2668. https://doi.org/10.1246/bcsj.73.2663.

[42] W.-H. Yang, V.F. Smolen, N.A. Peppas, Oxygen permeability coefficients of polymers for hard and soft contact lens applications, J. Memb. Sci. 9 (1981) 5367. https://doi.org/https://doi.org/10.1016/S0376-7388(00)85117-0.

[43] R. Witteck, B. Veith-Wolf, H. Schulte-Huxel, A. Morlier, M.R. Vogt, M. Köntges, R. Brendel, UV-induced degradation of PERC solar modules with UV-transparent encapsulation materials, Prog. Photovoltaics Res. Appl. 25 (2017) 409-416. https://doi.org/10.1002/pip.2861.

[44] R. Witteck, B. Min, H. Schulte-Huxel, H. Holst, B. Veith-Wolf, F. Kiefer, M.R. Vogt, M. Köntges, R. Peibst, R. Brendel, UV radiation hardness of photovoltaic modules featuring crystalline $\mathrm{Si}$ solar cells with $\mathrm{AlO} x / \mathrm{p}+$-type $\mathrm{Si}$ and $\mathrm{SiN} y / \mathrm{n}$ + -type Si interfaces, Phys. Status Solidi - Rapid Res. Lett. 11 (2017) 1700178. https://doi.org/10.1002/pssr.201700178. 
Fig. 1.- The three configurations of PV minimodule with integrated DS layers that are studied in this work.

Fig. 2.- Irradiance spectra of the $150 \mathrm{~W}$ xenon lamp of the climatic chamber used in this work.

Fig. 3.- EQE of the PV minimodule: (i) bare PV minimodule; (ii) with a DS layer encapsulated; (iii) with a DS layer faced down; and (iv) with a DS layer faced up in contact with the atmosphere. EQEs in the (a) $300-1200 \mathrm{~nm}$ and (b) $300-355 \mathrm{~nm}$ spectral ranges.

Figure 4.- Integrated increase in EQE values in the range $300-380 \mathrm{~nm}$ for the DS layers subjected to UV degradation in the climatic chamber.

Figure 5.- Linear degradation expected for the different DS layers on the PV minimodule and subjected to UV irradiation. 